\title{
Grupos minoritarios y acción positiva: las mujeres y las políticas de igualdad
}

\author{
Raquel 0 sborne
}

Universidad N acional de Educación a D istancia (U N ED )

\section{Resumen}

El texto trata de la desigualdad de la mujer frente al hombre en las sociedades avanzadas. Las acciones positivas para establecer la igualdad de oportunidades pretenden igualar las condiciones del punto de partida. Resultan insuficientes en muchos casos como medidas de discriminación positiva pero son un punto de partida crucial en el diseño de políticas de igualdad.

Palabras clave: mujer, género, acción positiva, políticas de igualdad sexual.

Abstract. M inority groups and positive action: women and equality policies

The text deals with woman's inequality in advanced societies. Positive actions in order to establish equal opportunities try to level life conditions. They are probably not adequate as positive discrimination measures but surely are the starting point for equality policies.

Key words: woman, gender, positive action, sexual equality policies.

\section{Sumario}

La definición de las acciones positivas

La «reacción» contra las acciones positivas

La igualdad en los resultados Razones de justicia

Para que las mujeres sean consideradas como susceptibles de medidas de acción o discriminación positiva tienen que haber sido previamente catalogadas como un grupo oprimido 0, más específicamente, como un grupo minoritario. ¿Cómo es posible que las mujeres, que numéricamente representan, en una sociedad como la nuestra, más del $50 \%$ de la población, puedan ser catalogadas como minoría? Para ello se ha de atender antes a la posición de subordinación social, política y económica de sectores de la población que a su número en sentido estricto. Así, los términos de «mayoría» y «minoría» des- 
criben más el poder de los grupos que su tamaño relativo. Colette G uillaumin, conspicua representante de la corriente de «feminismo materialista» francófona, lo expresa de la siguiente manera: «Por minoritarios entenderemos, no aquéllos que serían forzosamente menores en número, sino más bien aquéllos que en una sociedad están en estado de "menor poder", sea este poder económico, jurídico, político [... ]»¹. Subordinación histórica, carencia de poder y reconocimiento de la discriminación subsecuente serían requisitos imprescindibles para que a un grupo como a las mujeres se les reconozca el estatuto de «minoría».

Como expongo en un artículo publicado recientemente ${ }^{2}$, una tradición sociológica que arranca de Simmel, vinculada posteriormente a la pujante Escuela de Chicago de los años veinte y treinta y representada por autores como Robert Park o Louis W irth ${ }^{3}$, entre otros, fue la que comenzó a definir a las minorías por mor de la inferioridad del estatus y no por su envergadura estadística. $Y$ si bien el término fue en sus inicios aplicado a los grupos cuya problemática interesó vivamente a los miembros de aquella escuela - judíos, inmigrantes, negros- , esta forma de entender a las minorías fue la que permitió la aproximación sociológica entre las Ilamadas minorías étnicas y las mujeres. Posteriormente, ya en los años cuarenta y principios de los cincuenta, autores como Gunnar M yrdal, Everett $\mathrm{H}$ ugues y $\mathrm{H}$ elen $\mathrm{H} \mathrm{acker}^{4}$ efectuaron la conexión entre las minorías «históricas» y las mujeres. Fue quedando patente que las mujeres eran vistas como discriminadas por su adscripción a un master status o estatus principal - el ser mujer - al que se asignan determinados estereotipos en virtud de esa pertenencia al grupo de las mujeres. Ya no se trataba únicamente, pues, de un fenómeno vinculado a la mezcla de diferentes «razas» y culturas, sino relacionado con situaciones de amplio conflicto y cambio social, generadoras de una confusión en la identidad social de las personas adscritas a determinados grupos. La proliferación de acontecimientos

1. Guillaum IN , C olette (1992). «Femmes et théories de la société: Remarques sur les effets théoriques de la colére des opprimées». En G UILLAU M IN, Sexe, Race et Pratique du Pouvoir. París: C oté femmes, p. 219. Véase asimismo a este respecto: M AD o o LEN GERM AN N, Patricia; N Iebru G Ge-BrantLey, Jill (1993). «Teoría feminista contemporánea». En RITZER, G eorge, Teoría sociológi ca contemporánea, trad. M aría Teresa C asado Rodríguez. M adrid: M cG rawH ill, p. 353-409, esp. p. 358.

2. O SBORNE, Raquel (1996). «Son las mujeres una minoría?». I segoría, núm. 14, octubre de 1996, p. 79-93.

3. PARK, Robert (1928). «H uman M igration and the M arginal M an». American Journal of Sociology, 33, mayo de 1928, p. 881-893. W IRTH, Louis (1928). The Guetto. The University of Chicago Press. Posteriormente W irth precisaría más su pensamiento acerca de las minorías en el artículo «T he Problem of M inority Groups» (1945). En LINTON, Ralph (ed.). The Science of M an in the World Crisis. N ueva York: Columbia U niversity Press, p. 347-372, esp. p. 347.

4. M YRDAL, Gunnar (1944). An American D ilemma. Nueva York: H arper Brothers, p. 10731078; H u gues, Everett C. (1949). «Social Change and Status Protest: An Essay on the M arginal M an». Phylon, First Q uartet, vol. X, p. 58-65; M AYER H ACKER, H elen. «W omen as a M inority Group». Social Forces, vol. 30, octubre de 1951, p. 60-69. 
y tranformaciones que cristalizaron a partir de los años sesenta constituyó uno de esos períodos propicios para la aparición, no ya sólo del hecho de la discriminación - es decir, de sus aspectos «objetivos»- , sino también, lo que es más importante, de la conciencia de la misma - aspectos subjetivos- , factores ambos necesarios para que un grupo pueda ser clasificado como una minoría.

La primera cuestión - lo que hemos denominado aspectos «objetivos»y que se refiere a aspectos relacionados con el prejuicio y la discriminación en su aplicación a las mujeres, comenzó por entonces a ser estudiada por la sociología y la psicología social. Philip Goldberg, en un famoso trabajo sobre los prejuicios de las mujeres acerca de sí mismas, analizó la baja autoestima que mostraban las mujeres al calificarse, en igual dad de condiciones que los varones, de manera muy inferior ${ }^{5}$. M atina $\mathrm{H}$ orner, por su parte, expresó la forma en que las mujeres manifestaban «miedo al éxito» en sus estudios al vislumbrar las consecuencias negativas que aquél les podría acarrear - rechazo social, acusaciones de ser poco femeninas y cosas por el estilo- ${ }^{6}$. En parecidos términos se había manifestado años antes una conocida autora funcionalista, M irra Komarovsky, cuando descubrió que muchas buenas estudiantes «se hacían las tontas» con los chicos para no desafiar la expectativa cultural acerca de su inferioridad y no disminuir su atractivo ante éstos ${ }^{7}$. Algunos autores, que analizaron, junto a la de las mujeres, la problemática de la minoría negra, interpretaron los trabajos que acabamos de mencionar, amén de los nuevos datos por ellos investigados, como una calculada estrategia de supervivencia, como una dramaturgia que permitiría, llegada la ocasión, la movilización colectiva capaz de impulsar el cambio social, como así ha ocurrido en el caso de los negros y de las mujeres ${ }^{8}$. La conciencia colectiva de la discriminación - el segundo factor mencionado- comenzó por aquellos años sesenta y setenta con la aparición del movimiento feminista. La impulsión de medidas de acción positiva aplicables a este colectivo sólo fue ya una cuestión de tiempo.

5. Goldberg, Philip. «Are Women Prejudiced against Women?». Transaction, 5 de abril de 1968, p. 28-30.

6. H ORN ER, M atina S. (1968). Sex D ifferences in Achievement M otivation and Performance in Competitive and N on-C ompetitive Situations, tesis doctoral no publicada. Universidad de M ichigan. Cit. por Schur, Edwin M . (1984). Labeling Women D eviant. Gender, Sigma, and Social Control. N ueva York: Random House, p. 41.

7. Kom arovsky, M irra. «Cultural Contradictions and Sex Roles». American Journal of Sociology, vol. 52, noviembre de 1946, p. 184-189. Para críticas a los trabajos de H orner y Komarovsky, véase: T RESEM ER, D avid (1975). «Assumptions M ade About Gender Roles». En M ILLAN, M arcia; M oss Kanter, Rosabeth (eds.). Another Voice. Garden City, N ueva York: Anchor Books, p. 308-339.

8. W ARn ER, R. Stephen; W ellm An, D avid; Weitzm An, Lenore J. «T he H ero, the Sambo and the $O$ perator: Three C haracterizations of the $O$ ppressed». U rban Life and Culture, vol. 2, núm. 1, abril de 1973, p. 53-84. 


\section{La definición de las acciones positivas}

¿Cómo podríamos definir las acciones positivas? Como el establecimiento de medidas temporales que, con el fin de establecer la igualdad de oportunidades en la práctica, permitan mentalizar a las personas o corregir aquellas situaciones que son el resultado de prácticas o de sistemas sociales discriminatorios?. Al igual que lo sucedido con otras minorías, el reconocimiento sociológico como grupo susceptible históricamente de haber sufrido discriminación permite pensar en medidas correctoras que habrían de compensar pasados agravios. EI retraso en el logro de la igualdad entre los sexos derivaría del tradicional reparto de tareas y roles, fundamento de estructuras, actitudes y comportamientos perjudiciales sobre todo para las mujeres.

Cuando se habla de acciones positivas se está pensando que las políticas tradicionales conducentes a la igualdad de oportunidades, cuyo objetivo es la igualdad de condiciones del punto de partida, resultan insuficientes en situaciones o grupos en los que se parte de una desigualdad real sustantiva, por más que la igualdad formal se haya conseguido. ¿A qué nos referimos con desigualdad real sustantiva? A lo que Berit Âs comentaba acerca de que cuando dos grupos compiten por un mismo bien pero existe entre ellos una diferencia importante en cuanto al acceso a los recursos, el poder de los amigos, el tiempo disponible, los modelos de socialización y el control de la propiedad, por mencionar algunos importantes factores pero no los únicos, el grupo más fuerte ganará inevitablemente la competición de que se trate ${ }^{10}$. Por ello, si no queremos continuar reproduciendo la desigualdad aunque nuestra retórica no hable de otra cosa que de igualdad, para lograr una política efectiva en este terreno se nos aparecen dos vías complementarias: ampliar espacios de poder en todos los frentes para el grupo implicado así como, en un ámbito mucho más limitado, tratar de garantizar la igualdad en los resultados, que es lo específico de las acciones positivas. Q uizá convenga aclarar algo más este último aspecto.

Las acciones positivas, como bien sabemos, son una forma de diferenciación para la igual dad ${ }^{1}$. Pero para poder distinguir entre las diversas políticas encaminadas a este fin, deberíamos discernir entre objetivos y cuotas: mientras los objetivos marcarían un programa de acción positiva no cuantificada, las cuotas establecen una reserva de un mínimo garantizado de plazas, lograda por variados procedimientos. La verdadera acción positiva se hace con cuotas porque, o los objetivos son indicativos y no obligan real mente a adoptar medidas de acción positiva como las que aquí estamos comentando, o, si de veras obli-

9. CASAS, José I gnacio. Guía didáctica. M adrid: M inisterio de Asuntos Sociales (Instituto de la $M$ ujer), sin fecha, p. A-2 y A-5.

10. Äs, Berit. «W hich Positive Actions... at this Time in H istory?». Ponencia presentada en el I Congreso Internacional sobre la acción positiva para las mujeres. Bilbao, 27-29 de junio de 1990, p. 3.

11. RuIz M IgUel, Alfonso. «Discriminación inversa e igualdad». En V ALCÁRCEL, Amelia (comp.) El concepto de igualdad. M adrid: Pablo Iglesias, p. 77-93, esp. p. 79. 
gan, han de concretarse en cuotas, en resultados concretos, como señalábamos antes ${ }^{12}$.

\section{La igualdad en los resultados}

Cuando en los Estados Unidos se proclama en 1964 la ley a favor de los dere chos civiles (la Civil Rights Act), aplicable inicialmente sólo a los negros, se abre la puerta a las medidas de discriminación positiva. Q ue dicha aplicación se amplíe a otros grupos minoritarios, incluidas las mujeres, será una cuestión de tiempo mediatizado, sobre todo, por la presión que el colectivo de mujeres ejerza y de la oportunidad que las distintas administraciones estadounidenses vean en dichas políticas ${ }^{13}$. Posteriormente se extenderán, en mayor 0 menor medida, a todos los países del ámbito occidental, incluida España, algunas de cuyas peculiaridades comentaremos brevemente.

El momento en que se comienza a tener en cuenta «a igualdad en los resultados» se produce en los Estados U nidos en 1965 tras un discurso del presidente estadounidense Lyndon Johnson. En él se sostenía que la simple igualación de las reglas de la competición resultaba insuficiente para concurrir en igualdad de condiciones para quienes durante tantos años se habían encontrado en situa ción de desigual dad, en este caso la minoría negra a causa de la esclavitud. Se necesitaba una ayuda especial para cuyo concurso la «igualdad legal » debía ser sustituida por la «igualdad en los resultados». H acia el final de la administración Johnson las empresas que tuvieran más de cincuenta empleados y contratos con el gobierno por encima de una cantidad especificada debían de crear planes de acción positiva que dieran como resultado, y éste es el término clave, la contratación de un cierto número de empleados pertenecientes a minorías si querían seguir manteniendo relaciones contractuales con el gobierno ${ }^{14}$. La administración $\mathrm{N}$ ixon, interesada en no exacerbar los conflictos raciales que tanto conmovieron aquella sociedad durante los años sesenta, continuó con esta política, respaldada por la corte suprema ${ }^{15}$.

Aunque a partir de 1967 las mujeres estadounidenses fueron incluidas en estas normativas, la ley se desarrolló hasta 1987 de manera desigual y fue interpretada de manera ambigua en lo que a las mujeres concierne ${ }^{16}$. Ello se debía a que, mientras que la discriminación por razones de etnia 0 «raza» se aceptaba como un hecho dado, la discriminación por razones de sexo tenía que ser jus tificada. Tomó al menos veinte años hasta que una sentencia de la corte suprema la tuviera en cuenta para dar el espaldarazo sociojurídico a estas políticas en relación con las mujeres.

12. Ibídem, p. 81.

13. KeSSLER-H ARRIS, Alice (1994). «Feminism and Affirmative Action». En M ILLS, N icolaus (ed.). D ebating Affirmative Action. Nueva York: A D elta Book, p. 68-79, esp. 71; aunque M ills, en el mismo libro (p. 12), sostiene que esta ambigüedad duró sólo hasta 1971.

14. MILLS, op. cit., p. 6-8.

15. M ILLS. Ibídem, p. 9-10.

16. KESSLER-H ARRIS, op. cit. 
En España las principales medidas de acción positiva, en el sentido a que antes hemos aludido de las cuotas, se han aplicado sobre todo en el área de la participación política: son al gunos partidos políticos los que han comenzado a practicar estas medidas entre las filas de sus afiliados, como es de sobra conocido. En otros terrenos, en especial el laboral, no se han llevado a cabo medidas de acción positiva propiamente dichas aplicables a las mujeres como grupo, por mucho que se utilice profusamente el término ${ }^{17}$.

¿Cuáles podrían ser algunas de las razones que explican este fenómeno? Si nos atenemos al marco jurídico, la Constitución española resulta más avanzada en esta materia que la de otros países de nuestro entorno. Pero otros dos factores, cuya concurrencia se suele considerar necesaria para el fomento de este tipo de políticas de igualdad, se dan cita de manera mucho más endeble en España que en la mayoría de los países europeos y que en los Estados U nidos, con una mayor tradición democrática y mayores cotas económicas. Los factores aludidos son, de una parte, la menor incorporación de las mujeres al mundo laboral en nuestro país, y eso a pesar del notable incremento que se ha producido en los últimos años; y, de otra parte, la inexistencia de un movimiento feminista fuerte. Al no concurrir estos dos factores en la misma medida que en los otros países citados, no se han dado las condiciones propicias para la extensión de estas medidas en sus diversos ámbitos de actuación.

\section{La «reacción» contra las acciones positivas}

La inclusión de las mujeres en estas políticas, en particular las referidas al mundo del trabajo remunerado, puso sobre el tapete las conflictivas relaciones entre el papel de las mujeres en la familia y su plena participación en el mercado laboral. N ociones como el valor del mérito en conexión con las oportunidades laborales de las mujeres, o la validez de los derechos de los individuos por oposición a los de los grupos, fueron sometidas a reexamen. Atravesándolo todo aparecía la relevancia de las diferencias (0, más bien, las desigualdades) de género'8.

Estas cuestiones, entre otras, estuvieron presentes en la polémica sentencia del Tribunal de Justicia de la Unión Europea que tuvo lugar en otoño de 1995, en la que se declaró contraria a las leyes comunitarias una disposición del Estado de Bremen, en Alemania, y que me gustaría comentar a continuación brevemente. $D$ icha disposición establecía que, para que hombres y mujeres se repartieran al $50 \%$ los puestos en la función pública, una mujer tenía preferencia sobre un hombre si su grupo estaba infrarrepresentado. Pues bien,

17. En el terreno laboral contamos sobre todo con medidas de fomento del empleo: las empresas que contraten a personas con discapacidades, 0 a mayores de 45 años, 0 a mujeres, percibirán algún tipo de beneficio, pero no están obligadas a ello excepto en el caso de las minusvalías, donde la ley obliga a contratar, en empresas de más de cincuenta trabajadores, un $2 \%$ de discapacitados si las empresas son privadas, o un $3 \%$ si son públicas. Este objetivo parece que se cumple más o menos en el caso de éstas últimas y, en muy escasa medida, en la esfera privada. Fuente: C omisiones 0 breras, comunicación verbal.

18. KeSSLER-H ARRIS, op. cit., p. 72. 
siguiendo la disposición aludida, a igualdad de puntuaciones se había decidido conceder una plaza - lo cual suponía un ascenso- a la mujer aspirante, frente al varón que también concursaba. El aspirante recurrió al Tribunal de Justicia de la Unión Europea, el cual dictó sentencia a su favor. La sentencia consideró que la discriminación positiva de la mujer es ilegal cuando se aplica de forma «absoluta e incondicional » ${ }^{19}$.

Como sabemos, las leyes no se aplican en el vacío sino que se hallan muy relacionadas con el contexto. D esde una óptica feminista, lo más grave de la sentencia no sería tanto la estricta interpretación jurídica sino el mensaje que está transmitiendo, negativo para las mujeres, mensaje no casual en la Europa de los noventa ${ }^{20}$. Ciñéndonos, no obstante, a los términos de la sentencia, se podría discrepar de la misma si se entiende que la ley de Bremen no hablaba «de preferencia absoluta y automática de las mujeres, ya que la discriminación positiva sólo se aplica cuando se da igual dad de capacitación entre dos candidatos y si el grupo de la mujer está infrarrepresentado»²1. En este caso se había dado un empate en las calificaciones, de lo cual se deduce que resultaba obligada la adopción de algún otro criterio para elegir a alguno de los candidatos ${ }^{22}$.

Siempre que no entra en liza la discriminación positiva parece pensarse que los criterios de selección son justos y se hallan libres de valores culturales. Sólo cuando entran en juego las acciones positivas se esgrime la supuesta pureza de los criterios aludidos, que una política como la aquí comentada amenazaría con vulnerar. Pero una observación más cercana revela que las percepciones de las calificaciones basadas en el mérito fluctúan con el estatus social de la persona a evaluar $y$, en consecuencia, se encuentran ligadas a la pertenencia a un grupo ${ }^{23}$. Como señala Richard Wasserstrom, resulta casi un lugar común la proclividad que muestran quienes se encargan de contratar a otras personas hacia la selección de aquéllas que les resultan más afines, entre otras por razones de género e incluso por la inercia que da la costumbre ${ }^{24}$. No en vano se habla de discriminaciones indirectas que no resultan necesariamente de actitudes discriminatorias adoptadas de forma intencionada, sino que provienen básicamente de hábitos sociales ${ }^{25}$. En clave feminista lo conceptualizaríamos como una muestra más de los «pactos entre varones» que diría C elia Amorós, por los cuales se acaban valorando factores que no designan necesariamente a la

19. El País, 28 de octubre de 1995.

20. El País, 26 de octubre de 1995.

21. Alberdi, Cristina, en declaraciones a El País, ibídem.

22. Ruan o Rodríguez, Lucía; Pérez del Río, M aría Teresa. «La difícil instrumentación dela igualdad». El País, 2 de noviembre de 1995.

23. Eberh ARDT, Jennifer L.; FISKE, SUsan T. «Affirmative Action in Theory and Practice: Issues of Power, Ambiguity, and G ender Versus Races. Basic and Applied Social Psychology, número especial: «Social Psychological Perspectives on Affirmative Action». TURNER, M arelene, E.; PratKanis, Anthony R. Guest Editors, p. 201-220, esp. p. 205.

24. W ASSERST ROM, Richard A. (1980). Phil osophy and Social Issues. Indiana: University of N otre D ame Press, p. 70-71.

25. CASAS, op. cit. 
persona más cualificada sino a la más afín, pues entran en consideración otros aspectos «extracurriculares», tanto más cuanto más elevada sea la posición disputada en términos de prestigio o poder. Las acciones positivas tenderían precisamente a corregir estos defectos.

$\mathrm{N}$ os topamos, en cualquier caso, con una de las razones que se esconden tras el llamado «techo de cristal», cuyas bambalinas no siempre se ponen de manifiesto de forma suficiente. Los científicos sociales, por su parte, al aceptar la tradicional noción de la selección como independiente de la pertenencia a un grupo prestan escasa atención a la forma en que el género beneficia a los varones ${ }^{26}$.

En cuanto al segundo aspecto que queríamos comentar aquí, el citado tribunal europeo opina que es injusto que al guien pueda sufrir una merma en sus derechos individuales por razón de sexo, aunque el objetivo sea beneficiar a un grupo tradicionalmente desfavorecido ${ }^{27}$. N os enfrentamos aquí con una crítica habitual a las acciones positivas cuando se afirma que en ellas no se contempla a las personas como individuos sino como instrumentos de políticas sociales $^{28}$. C on todo lo que de discutible tenga este argumento, tras él se produce el ocultamiento del atributo de género en las decisiones cuando de los varones se trata (o de los blancos si lo referimos a la «raza»), «prejuicio» que se esgrime cuando se trata de mujeres (o de los negros), es decir, cuando se trata de «minorías». En el primero de los casos no se está teniendo en cuenta la forma en que el estatus de grupo, ligado al género y a la «raza», influye en los resultados. C omo ya comentábamos en otro lugar, «esta visión ignora las disparidades en el poder de los diferentes grupos, divergencias que hace aparecer la distribución de recompensas como ostensiblemente "neutras" y no ligadas a la "raza" y al género, excepto cuando se trata de los sectores o grupos más desfavorecidoss ${ }^{29}$.

Q uienes no tienen en cuenta estos factores objetan, según comenta R uiz $M$ iguel, «que este tipo de política no considera la individualidad de las personas, hasta el punto de que las toma como instrumentos para la obtención de fines sociales. La fuerza de este argumento depende de la concepción que se tenga de grupo social y de la relación que se suponga entre los grupos y los individuos que los componen ${ }^{30}$. Ello enlaza con las polémicas contemporáneas entre posiciones individualistas y posiciones de corte comunitarista. Frente a esta última perspectiva cabe argumentar que no somos meros apéndices de un grupo, de una comunidad, y que no es cuestión de anular la individualidad por una instancia superior, por «o social», por mor de un loable fin igualitario. Pero al mismo tiempo, convendría también rebajar el alcance de un estricto individualismo a lo Robert N ozick, que no tendría en cuenta que ciertos individuos se ven discriminados por su pertenencia a un grupo y, por

26. EBERHARDT, op. cit., p. 206.

27. El País, 18 de octubre de 1995.

28. RUIZ-MIGUEL, op. cit., p. 89.

29. EBERH ARDT, op. cit., p. 203. En «Son las mujeres una minoría?». I segoría, op. cit.

30. RuIZ-MIgUeL, op. cit., p. 89. 
ende, la superación de tal injusticia deberá ser hecha también desde una óptica de grupos ${ }^{31}$.

M uchos de los que se manifiestan en contra de las acciones positivas suelen expresar la queja de que van «más allá de una promoción de la igual dad de oportunidades», como recoge la propia sentencia ${ }^{32}$. Parece olvidarse que, como vimos más arriba cuando el discurso del presidente Johnson, ése es justamente el fundamento de este tipo de políticas: la consideración de que la promoción de la igualdad de oportunidades, aplicable a todos por igual, resulta contraria al principio de igual dad cuando se aplica a personas pertenecientes a grupos que no se encuentran en situación de igualdad real, sustancial y no sólo formal. Q uienes se oponen a las políticas aquí discutidas tienen muy clara la contraposición entre los dos términos, apostando siempre por la igualdad de oportunidades - aplicable a los individuos - y renegando de una política que pone el énfasis en la igual dad de los resultados - aplicable a determinadas personas a causa de su pertenencia a ciertos grupos ${ }^{33}$.

No tener en cuenta la relación entre la discriminación de las mujeres y su pertenencia al colectivo femenino es aplicar la ley del embudo, según Ruiz M iguel. Significa, según este autor, «tratar como grupo a las mujeres cuando se las discrimina al modo tradicional» - es decir, discriminarlas no tanto como individuos sino por su inevitable vinculación al grupo de las mujeres- y, sin embargo, supone pensar a los hombres estrictamente como individuos en los casos en que «se intenta igualar a las mujeres mediante la discriminación positiva» 34 . Con esto se está olvidando o negando que en realidad ellos han sido favorecidos desde siempre por su pertenencia al grupo de los varones, como comentábamos antes, pero eso no se ha querido ver nunca: es una realidad invisible sólo puesta en primer plano cuando las mujeres se han negado a aceptar, no ya sólo históricas discriminaciones, sino también la perpetuación, a menudo impulsada por las inercias alimentadas por la costumbre, de estos mecanismos.

Estetipo de políticas se verá favorecido, por lo general, por quienes, como grupo, se beneficien de ellas ${ }^{35}$. Conviene tener presente que, habitualmente, la competición se efectúa en un contexto de recursos escasos, tal y como acontece con los puestos de trabajo. En contra de que las mujeres se beneficien de estas políticas se esgrime con frecuencia que, en muchos casos, necesitan el soporte económico masculino para sobrevivir. Si bien esto es cierto, no lo es menos que precisamente la razón estriba en esa historia, extendida hasta el presente, de exclusión generalizada de la esfera de lo público, en especial de lo laboral; cuando se efectúa la integración en el mundo del trabajo remunerado, ésta se lleva a cabo en un contexto de mercado laboral segregado y jerarquiza-

31. Ibídem, p. 89.

32. El País, 26 de octubre de 1995.

33. MILLS, op. cit., p. 17 y 31.

34. RUIZ-M IGUEL, op. cit., p. 89-90.

35. MILLS, op. cit., p. 33. 
do entre los sexos, así como de escasa diversidad de oferta laboral para las muje res. A ello se suma su discontinua dedicación a este mercado a causa del mandato social de la maternidad y a su más frecuente expulsión del mismo debido a la mayor precariedad de sus empleos. Todo ello ayuda a explicar la situación de dependencia económica respecto de los varones por parte de muchas mujeres. Cuando se habla, además, de soporte económico masculino se sobreentiende - pero no se explicita muchas veces- la esposa «y» los hijos: es mucho más plausible que una mujer sola logre autofinanciar su vida, a pesar de las limitaciones anteriormente mencionadas, que una mujer con hijos a su cargo - precisamente por las dificultades a que nos hemos referido, mucho más complejas de lo que aquí estamos dando a entender por motivos de espacio.

Aunque éstas y otras razones, como sabemos, avalen esta situación de dependencia, los defensores de las políticas de acción positiva sostienen, además, que hoy en día el número de mujeres que mantiene un hogar va en aumento. Es decir, se trata de equilibrar un argumento con otro. D e hecho, pudiera darse $\mathrm{e}$ caso de que ambas situaciones fueran, numéricamente hablando, equiparables, es decir, que hubiera igual número de mujeres - madres de familiaeconómicamente dependientes que de mujeres con responsabilidad exclusiva en las cargas familiares. Podría igualmente no ser ésta la situación, y que la balanza se desequilibrase hacia uno u otro lado. Pero al margen del resultado de estos cálculos, quienes defienden estas políticas continúan señalando la mirada de razones que, a su juicio, las siguen haciendo defendibles:

- M ientras el trabajo de las mujeres - remunerado y no romunerado- se vea como menos importante, estará peor pagado que el de los hombres.

- M ientras se las siga viendo como las principales responsables de todo lo que concierne a la familia, difícilmente se entenderá con todas las consecuencias que ellas tienen todo el derecho a la incorporación plena al mundo del trabajo remunerado; por lo tanto, difícilmente se apoyarán socialmente las políticas que fomentan la plena incorporación laboral de las mujeres.

- M ientras se siga interpretando la actividad de la reproducción social como femenina, y por lo tanto, con menor prestigio que la que efectúan los varones, éstos se negarán a llevarla a cabo.

- M ientras los hombres no asuman los asuntos familiares con plena responsabilidad, las mujeres no se podrán librar de la doble jornada laboral - doméstica y extradoméstica - y siempre saldrán perdiendo porque no podrán satisfacer adecuadamente las responsabilidades familiares y las laborales. Esta doble jornada refleja, en cualquier caso, la desigual dad estructural de esta situación con respecto de la que viven los hombres ${ }^{36}$. $\mathrm{N}$ i que decir tiene que nos estamos refiriendo a estructuras patriarcales, y por lo tanto, asumidas por la mayoría de los varones pero igualmente por no pocas mujeres. 


\section{Razones de justicia}

A juicio del abogado del alto tribunal de la Unión Europea, la lucha contra la discriminación de las mujeres se debe realizar, no mediante medidas preferenciales en el puesto de trabajo sino «con medidas relativas a la organización del trabajo y, en particular, al horario de trabajo, las estructuras destinadas a la infancia y otras medidas que permitan conciliar las obligaciones familiares con las laborales, ${ }^{37}$ - para los dos sexos, añadiríamos desde aquí- . Es decir, mientras no exista conciencia de la conveniencia de una transformación de las relaciones entre las obligaciones laborales y familiares en un sentido más igualitario por y para ambos sexos, cual quier política tendrá un al cance claramente limitado. Pero, entretanto, los objetivos organizativos a los que apunta el citado abogado no tienen por qué excluir medidas de acción positiva si de justicia estamos hablando. Los costes, en efecto, pueden ser altos en términos de los resentimientos que causa entre quienes no son incluidos en al guno de los grupos favorecidos por las mismas. Pero, se contraargumenta, puede entenderse como de mayores consecuencias negativas un sistema que permite que las desigualdades del pasado continúen en el presente ${ }^{38}$. En consecuencia, como declaraba un juez estadounidense, «para lograr sobrepasar el sexismo debemos antes que nada tener en cuenta el sexo ${ }^{39}$.

Si nos halláramos en una situación de pleno empleo las soluciones no presentarían las dificultades que una tónica de desempleo crónico manifiesta. El día a día nos muestra, más bien, la competición entre grupos, algunos de cuyos miembros duplican su pertenencia, por un conjunto de recursos escasos. La apelación a la solidaridad antes que a la competencia se hace cada vez más necesaria, pero siempre que tratemos de romper planteamientos paternalistas de «nosotros» $\mathrm{y}$ 《ellos». En el caso de las mujeres, dichos planteamientos pierden, no obstante, parte de su significado en la medida que todas somos, en cierta medida, «diferentes» en el sentido dado a la definición de las minorías.

Frente al faccionalismo y la fragmentación - resultado posible de la constatación de la diversidad postmodernista - cabe fomentar una amplia política de alianzas que ponga coto a la desigualdad, origen de nuestra «diferencia».

M encionaba antes el mensaje negativo que una sentencia de esta índole transmitía a las mujeres. En efecto, algunos de los vientos que soplan no son los más favorables para este tipo de políticas. En los Estados U nidos, la Administración Clinton se pronunció en 1996 por una limitación drástica de las acciones positivas, mientras que los republicanos proponían su prohibición, sin mayores matices ${ }^{40}$. En las mismas fechas, la Unión Europea, sin embargo,

37. El País, 18 de octubre de 1995.

38. RUIZ-M IGUEL, op. cit.

39. BLACKM Un, Juez H arry. Citado por N icolaus M ills: «ntroduction: To Look Like America». En M ILLS, op. cit., p. 29.

40. El País, 11 de marzo de 1996. 
no sólo era partidaria de seguir fomentando las acciones positivas, sino que además se mostraba decidida a reforzar este tipo de mecanismos, no sólo por razones históricas de discriminación, sino también por la ardua situación presente, de previsible endurecimiento en el futuro. En España, al menos hasta parecidas fechas, desde las instancias oficiales se manifestaba una cierta proclividad hacia este tipo de políticas, como los planes de igualdad de oportunidades atestiguan y la publicidad institucional del Instituto de la M ujer proclamaba a bombo y platillo41. Los datos que se manejan para apoyarlas hablan por sí solos: según la Confederación Internacional de Sindicatos Libres, las mujeres son las primeras víctimas de la mundialización de la economía, que ha entrado en una descarnada competitividad internacional en detrimento de los derechos de los trabajadores y, sobre todo, de las trabajadoras, en un contexto en el que las mujeres no sólo cobran menos sino que, además, trabajan más que los hombres en todos los países del mundo ${ }^{42}$. Es decir, razones de justicia social avalan la defensa de estas políticas. Pero como muchos otros factores entran en juego, veremos lo que el futuro nos depara. Por lo menos se ha conseguido que las mujeres, consideradas como minoría en el sentido sociológico del término, hayan dejado de estar en los márgenes de las preocupaciones de las ciencias sociales para situarse en el centro de las mismas. Q ue las mujeres experimenten en su vida cotidiana estas transformaciones sería el siguiente paso. En eso estamos.

41. Véase en este sentido la publicidad aparecida en EI País, 8 de marzo de 1996, p. 27, en la que, abarcando toda la página, se veía una silueta femenina y dos leyendas: «L as mujeres avanzan», se supone que gracias a las «medidas de acción positiva», escrita con letras un poco más pequeñas.

42. El País, 8 de marzo de 1996. 